Case t.-Mrs. A. Delicate health, occasional prostrating nervous headaches, lasting two or three days. Suffers much from neuralgia. Both antipyrin and antifebrin in full doses relieve the headache, but even a single dose of 5 grs. of antipyrin produces distress in the region of the heart, and makes its beat irregular. Antifebrin produces no such feeling.

Case 2.-Mrs. B. Delicate health, but stronger than the preceding. Persistent nervous headaches of two or three days' duration often. Relieved by both remedies. No trouble felt from either.

Case 3. Mrs. C. Has good health; never sick except with nervous headache, which is hereditary and lasts two or three days. No heart trouble from either remedy. Antifebrin makes no perceptible impression. Antipyrin gives almost instant relief. This is the first remedy this lady has found that would diminish her suffering. Nervosanguine temperament.

Case 4.-Mrs. D. Usually good health, but often suffers with intense nervous headaches, lasting from twelve to forty-eight hours. Antifebrin produces no beneficial effect. Antipyrin gives relief. Like the preceding, nervo-sanguine temperament. All these patients have borne children.

Case 5.- Miss E. Sister of the preceding patient and of the same temperament. Is somewhat rheumatic, sometimes having slight muscular rheumatism, especially intercostal rheumatism. Often suffers with excruciating nervous headache. Has never used antipyrin. Antifebrin acts quickly.

The return of the headache in none of these cases can be traced to the menses, and they have no regular periodicity.

\section{LAPARO'TOMY FOR THE REMOVAL OF A FIBRO-MYOMA.}

Read before the Medical Society of the District of Columbia, March 27. 1888 .

BY P. J. MURPHY, M.D., OF WASHINGTON, D. C.

Nellie B., white, single, æt. 26, was admitted to the Columbia Hospital for Women November II, I887. Previous to the summer of I $886^{\circ}$ she had good health. At that time noticed a swelling in right side which gradually increased and troubled her a great deal. Menses have recurred regularly, lasting from four to seven days, without pain. At time of admission complained of being restless at night, of frequent smothering sensations and severe headaches. No trouble in voiding urine. Appetite and general condition good. Examination November I 2 revealed a hard pearshaped tumor of about the size of a head of a child 2 years old, extending from the brim of the pelvis upward nearly to the umbilicus, and freely movable laterally. By vagino-abdominal examination the uterus seemed to be slightly connected with the growth; cervix uteri somewhat shortened and soft. Diagnosis of a fibro-cyst of the uterus was made. Menstruated November I 9 to 28 ; flow profuse but not alarming. On December I was examined under ether and the growth was found to be freely movable in every direction except upward, and now quite reached the umbilicus. Body of uterus seemed to be displaced forward and to the right side and somewhat enlarged and hard. Some glairy mucus in cervical canal.

At a consultation of the Advisory Board of the hospital December 3 no definite conclusion was reached. From December 28 to February 22 electricity was applied daily (except during menstruation), about ten cells of a McIntosh galvanic battery being used, which gave as strong a current as the patient could bear without pain or too rigid contraction of abdominal muscles. Although the battery was faithfully tried the growth gradually increased, and at the time when electric treatment was suspended it reached a point about 2 inches above the umbilicus. Patient was discouraged with electricity and requested that the growth be removed by operation. At a second consultation of the Advisory Board February 29 it was decided to operate. Laparotomy was performed in the presence of Drs. Morgan and Head, of the Advisory Board, Dr. Linthicum, of Maryland, who formerly attended the patient, and Drs. Boyce and Sweetman, of house staff. An incision about 5 inches long was made in the median line midway between the umbilicus and symphysis pubis. When the growth was reached a number of large blood-vessels were seen upon its surface. It now seemed to be a continuation of uterus upwards, no line of junction being noticeable. A few smaller but harder growths were attached near the vaginal roof; one of which had probably been mistaken for the displaced uterus. Some of the gentlemen present felt the growth and thought that its contents were fluid. A trocar was inserted, but no fluid escaped. The incision was extended 2 inches upward to allow extraction of the tumor. When drawn out of the cavity it was found to embrace the uterus on every side, and its removal was agreed to.

\section{MEDICAL PROGRESS.}

Methylene AS AN ANeSTheTIC.-Dr. WM. $H$. DAY says that it is a mystery to him that an anæsthetic so safe and effectual as methylene should not have been more generally employed by the profession. A few deaths have been imputed to it, it is true, and occasional deaths will occur from any anæsthetic, however carefully administered, but notwithstanding, I think, methylene occupies in point of safety a first-class position. Some years ago the late Mr. Peter Squire asked me whether I considered methylene pos- 